\title{
Aspectos bioéticos de trabajos de investigación odontológica evidenciados en revistas cientificas.
}

\author{
Bioethical aspects of works research \\ odontologic evidenced in scientific journals.
}

Aspectos bioéticos das trabalhos

da investigação dental mencionados

nos periódicos científicos.
Olga Leonor Ariasgago

Directora de Proyecto de Investigación. Profesora adjunta de la Cátedra de

Odontología Legal.

E-mail: lariasgago@odn.unne.edu.ar

Natalia Inés Marasso Spaciuk Becaria de Investigación. Docente Auxiliar de Primera de la Cátedra de Odontología Legal.

E-mail: nataliamarasso@hotmail.com

José Robeto Rivarola

Sub Director de Proyecto de Investigación.

E-mail: jrivarola@odn.unne.edu.ar

Lugar de trabajo Facultad de Odontología.

Universidad Nacional del Nordeste Av. Libertad 5450 - 3400 - Corrientes.

Tel/fax: 03794457992

\section{Resumen}

La bioética en la investigación aspira a lograr que la ciencia esté al servicio del hombre. Este trabajo pretende analizar el tratamiento explícito de requisitos bioéticos en publicaciones de investigaciones odontológicas que involucran seres humanos. Se realizó un estudio descriptivo transversal sobre una muestra de 100 artículos. Se estudió la revisión por un comité de ética, consentimiento informado, justificación del estudio, selección de la muestra y criterios éticos en las normas de publicación. El $89 \%$ de los artículos no expresa haber sido evaluado por un comité y $68 \%$ no menciona haber realizado el consentimiento informado. El $91 \%$ de los estudios detalla los beneficios de la investigación y el $67 \%$ explica la metodología empleada para seleccionar a los sujetos. En el $67 \%$ de las revistas analizadas no se advierte una normativa bioética expresa. Este trabajo reflexiona sobre la necesidad de explicitar en las publicaciones los aspectos bioéticos en toda investigación en seres humanos.

\section{Palabras Clave}

Bioética, investigación en humanos, publicaciones.

\section{Abstract}

The bioethics research aims to make science at the service of man. This paper analyzes the treatment explicit of bioethical requirements in odontology publications research involving humans. A descriptive cross-sectional study on a 
sample of 100 items was conducted. Review by an ethics committee was studied, informed consent, justification of the study, sample selection criteria and ethical standards from rules of publication. The $89 \%$ of the items not express have been evaluated by a committee and $68 \%$ not mention to have made the informed consent. The $91 \%$ of the studies details the benefits of research and $67 \%$ explain the methodology used to select individuals. In $67 \%$ of the analyzed journals are not warned bioethics rules are required. This paper reflects on the need to make explicit in the publications, the bioethical requirements in all research involving human subjects.

\section{Keywords}

Bioethics, research on humans, publications.

\section{Resumo}

Bioética pesquisa tem como objetivo a ciência tornar a serviço do homem. Este artigo analisa o tratamento explícito de requisitos bioéticas en as publicações de dentales pesquisa envolvendo seres humanos. Um estudo descritivo transversal com uma amostra de 100 itens foi conduzido. Revisão por um comitê de ética foi estudada, consentimento informado, justificação do estudo, seleção da amostra e os critérios de éticos das normas de la publicação. Um $89 \%$ dos itens não foram avaliadas por uma comissão e $68 \%$ nao menciono ter feito a consentimento informado. Em um $91 \%$ detalham os benefícios da pesquisa e $67 \%$ explica metodologia utilizada para selecionar os indivíduos. Em $67 \%$ das revistas analisadas não a avisados expressamente as regras bioéticas. Este artigo reflete sobre a necessidade de tornar explícito na publicações, bioéticas questões em todas as pesquisas envolvendo seres humanos.

\section{Palavras chave}

Bioética, pesquisa em humano, publicações.

\section{Introducción}

La bioética en la investigación, no sólo abarca una serie de normas que intentan proteger a los sujetos de los excesos de la ciencia o tratan de impedir o limitar el desarrollo propio de ésta, sino que pretende lograr que la ciencia esté al servicio del ser humano. Es decir, que exista una relación creativa y respetuosa donde el investigador realice proyectos válidos, los sujetos estén interesados en participar y la sociedad obtenga resultados que contribuyan a tener una medicina más efectiva y eficiente a fin de mejorar la calidad de vida del humano'. La conducta ética del investigador requiere, en primer lugar, de la educación en temas de bioética para que crezca el razonamiento moral; el crecimiento de la capacidad para identificar dilemas éticos o puntos de conflicto; de la comprensión o razonamiento de los mismos, y del uso de principios éticos reconocidos a fin de encontrar conclusiones que permitan la toma de decisiones moralmente válidas $^{2}$. Los fines de la investigación o de la ciencia no pueden prevalecer sobre los del individuo, porque la ciencia está al servicio del hombre y no a la inversa ${ }^{3}$.

\section{La investigación en seres humanos y la Bioética}

La humanidad ha desarrollado principios éticos para controlar la investigación luego de haberse hecho público una serie de episodios denigrantes. Ejemplo de ello son los estudios, realizados al amparo del Proyecto Manhattan, en el cual se utilizaron mujeres embarazadas y sus fetos, enfermos desahuciados, recién nacidos, moribundos y ancianos que bajo engaño fueron utilizados para investigar los efectos de las radiaciones, fueron inyectados con dosis elevadas de isótopos o los ingirieron con alimentos sin tener conocimiento del material radiactivo que se introducía en sus cuerpos ${ }^{4}$. Otro suceso fue la investigación sobre el curso natural de la sífilis, utilizando campesinos negros analfabetos de Alabama (USA), a quienes bajo engaño y después de haberles hecho el diagnóstico de la enfermedad, se les privó de tratamiento con antibióticos, durante casi 40 años, mientras se les iban estudiando su deterioro físico; así como la incidencia de sífilis congénita en sus hijos y el grado de contagio, a otros miembros de la familia, quienes tampoco recibieron tratamiento.

La historia de las Declaraciones Medicas, comenzó al terminar la Segunda Guerra Mundial, 
cuando se fijaron normas sobre los requisitos necesarios para llevar a cabo estudios experimentales en personas; después de comprobar que muchos prisioneros, soldados o civiles, fueron utilizados en investigaciones clínicas de manera violenta, sin información, sin consentimiento y sin ningún respeto, por su condición de seres humanos; como si fueran simples animales de laboratorio ${ }^{5}$.

Es así, que en 1947 se elaboró el Código de Nüremberg ${ }^{6}$ que establece 10 normas que los profesionales de la salud deben respetar cuando llevan a cabo experimentaciones con seres humanos. La primer norma, establece que el "Consentimiento voluntario de los sujetos humanos es absolutamente necesario", así como la reducción del riesgo y del daño al mínimo, la proporción favorable del beneficio respecto al riesgo, idoneidad de las calificaciones de los investigadores, lo apropiado de los diseños de investigación y la libertad del participante para retirarse en cualquier momento de la investigación ${ }^{7}$.

En 1964 se promulgó la Declaración de Helsinki, donde se introducen nuevos elementos a considerar relacionados con el bienestar del individuo; el cual debe prevalecer sobre los demás intereses. Abarca además el tema de las poblaciones con autonomía disminuida e introduce el uso de consentimiento por escrito y establece que todas las investigaciones con seres humanos deben ser evaluadas y aprobadas por un comité de ética. Asimismo determina que "los informes sobre investigaciones que no se ciñan a los principios descritos en esta declaración no deben ser aceptados para su publicación"”. Esta Declaración ha sido revisada en por varias Asambleas Médicas Mundiales diferentes ${ }^{9} \mathrm{y}$, con sus modificaciones posteriores, constituyó el cuerpo básico de principios que fueron incorporados desde entonces a las numerosas Normas de Investigación que han surgido en los diferentes ámbitos de la investigación biomédica ${ }^{10}$.

En 1978 se redactó el Informe Belmont, cuyo contenido más importante se refiere a los principios éticos fundamentales que debe regir la experimentación con seres humanos además introduce una distinción entre la investigación y la práctica médica".

Diego Gracia ${ }^{12}$ describe la evolución de la experimentación clínica a través del tiempo re- lacionado con las significancia ética de cada pensamiento y divide en tres periodos. El primer periodo abarca desde los inicios de la medicina hasta el año 1900 a la que denominó "medicina basada en la intención". Dicha etapa se caracterizaba por considerar a la investigación clínica moralmente aceptada, sólo si su objetivo principal era el de beneficencia y sólo "por accidente" o secundariamente producía algún conocimiento científico. El segundo periodo (1900-1947) es llamado "medicina de la evidencia”, caracterizándose porque aparece el diseño metodológico. En el tercer periodo (1947 hasta nuestros días), los abusos continuaron y crecieron, constatándose hechos aberrantes. Se llega a la conclusión que la autorregulación del investigador era insuficiente, de allí que esta etapa es clave en la historia de las conductas éticas en las experimentaciones clínicas; pues la humanidad tomando conciencia de las implicancias, comienza a elaborar distintos documentos que ponen límites a la experimentación con seres humanos.

También hay que señalar que en numerosas experiencias con seres humanos impera, no una actitud de agresivo desprecio hacia ellos, sino un patente descuido y falta de sensibilidad moral, que pone en riesgo la propia vida de las personas. Muchos científicos están convencidos que es perfectamente aceptable y tolerable cometer faltas éticas, si está en juego un conocimiento científico, considerando a éste un interés superior.

Vélez Correa afirma que en la investigación con seres humanos debe tenerse en cuenta, ante todo, los tres principios fundamentales de la ética natural: autonomía, justicia y beneficencia y resume las normas éticas que deben regir la experimentación biomédica en seres humanos: I. El beneficio de la humanidad no debe anteponerse al daño que se haga a una persona, 2. El beneficio de la investigación debe ser mayor que los riesgos de daño que se deriven del desarrollo de la misma, 3. Se debe salvaguardar durante la investigación no solo la autonomía y la vida de los sujetos en los cuales se experimenta, sino también su integridad física, psíquica y social,4. Debe obtenerse un consentimiento pleno e informado del sujeto que va a participar en la investigación, 5 . Si se mezcla la prestación de servicios con experimentación, debe explicárse- 
le claramente al paciente para que éste pueda aceptar o rechazar libremente el servicio, 6. El sujeto en el cual se realiza una investigación participación tiene derecho a retirarse de ella en cualquier momento, 7. La experimentación debe hacerse primero en vitro y en animales antes que en humanos, 8. Los informes de la investigación deben ser ceñidos a la verdad, tanto en lo relacionado con los materiales y métodos como con los resultados.

De ahí que el científico moderno debe preguntarse no sólo ¿Puedo hacer esto?, sino: ¿Debo hacerlo? Esta segunda respuesta no la puede dar sino el científico con criterio ético ${ }^{13}$.

En las dos últimas décadas surgió entre los editores de revistas científicas una preocupación por determinar orientaciones estandarizadas para la elaboración de artículos que sean sometidos a publicación. Es así que se formó el Comité Internacional de Editores de Revistas Médicas, el cual edita los Requisitos Uniformes para Manuscritos Sometidos a Revistas Biomédicas (RUMSRB). Las recomendaciones de los RUMSRB hacen referencia explícita a la Declaración de Helsinki y a comités responsables para la experimentación humana. De esta manera, se solicita a las revistas que concuerdan con los RUMSRB, que el documento sea citado en sus Instrucciones a los Autores. Actualmente, gran parte de las revistas científicas del área de la Odontología hacen referencia a aspectos éticos de la investigación en seres humanos en sus Normas para Autores.

Trajano Sardenberg y colaboradores, pertenecientes a la Universidad Estadual Paulista Julio de Mesquita Filho UNESP (San Pablo, Brasil) indagaron los aspectos bioéticos en las publicaciones científicas, utilizando como muestra de su estudio la sección de "Instrucción para los Autores" de 139 revistas científicas brasileñas de medicina, biomedicina, enfermería, odontología y ciencias generales; analizándolas teniendo en cuenta la orientación ética en la investigación en seres humanos. Estas revistas fueron agrupadas según su especialidad médica. El análisis se basó en relación a los aspectos éticos de las orientaciones que contenían estas publicaciones, clasificándolas en seis categorías: I) Comisión o Comité de Ética (aquellas que hacen referencia a la necesidad de aprobación y/o análisis de los trabajos por un comité de ética), 2) Declaración de Helsinki (no hay referencia a comité de ética pero citan a esta declaración), 3) Consentimiento del Paciente (sólo hacen referencia a este aspecto ético), 4) Principios, Normas, Estándares Éticos, 5) Requisitos Uniformes (hacen referencia a requisitos Uniformes para Manuscritos Sometidos a Revistas Biomédicas, propuesto por Comité Internacional de Revistas Médicas) y 6) Sin orientación ética. Los resultados más significativos fueron la obtención de un bajo número de revistas que hacen referencia a los aspectos éticos en la investigación $(20,8 \%)$, dentro de las cuales, gran parte requería que la información sobre estos aspectos éticos sea incluida dentro del artículo $(57,8 \%)^{14}$.

No se hallaron estudios específicos que describan la evidencia de los requerimientos bióeticos en las publicaciones que traten sobre investigación en Odontología realizadas en individuos.

Este trabajo pretende analizar el tratamiento explícito de los requisitos bioéticos en las publicaciones de investigaciones odontológicas que involucran seres humanos.

\section{Objetivo General}

Determinar el tratamiento explicito de los requisitos bioéticos en las publicaciones de investigaciones odontológicas que involucran seres humanos.

\section{Objetivos Específicos}

- Analizar los requerimientos bioéticos tenidos en cuenta en las instrucciones para autores de las publicaciones.

- Ponderar la mención explicita de los comités de bioética en los trabajos de investigación y valorar la descripción del Consentimiento Informado que aparece en los artículos científicos.

- Establecer la existencia de una fundamentación valida que justifique la investigación en los aspectos de la ecuación riesgo - beneficio. 


\section{Materiales y Métodos}

Este trabajo corresponde a un estudio descriptivo de corte transversal. La muestra consistió en 100 artículos de investigación en Odontología que involucren seres humanos correspondientes a formatos on-line (utilizando buscadores virtuales (Latindex y Scielo), e impreso. Este último radicó en artículos de revistas de Odontología disponibles en la Biblioteca de la Facultad de Odontología de la Universidad Nacional del Nordeste.

Los criterios de inclusión que se tuvieron en cuenta fueron: revistas de odontología editadas en castellano, que contengan algún artículo indexado de investigación en humanos, que sea texto completo, empleando un diseño tanto experimental como no experimental; que la edición de la misma ofrezca al menos un ejemplar anual, y que la revista estuviera editada en el periodo comprendido entre los años 2000 a 2010.

Se encontraron vía online, tres revistas nacionales y siete extranjeras y en formato impreso, cuatro nacionales que cumplían con los mencionados criterios de inclusión.

Para la selección de la muestra se optó por el primer artículo del primer número y volumen de cada año, de cada revista seleccionada de la forma descripta anteriormente.

Se armaron grupos, estableciéndose las siguientes variables independientes: Periodos (2000/2005 y 2006/2010), Procedencia (Argentinas y Extranjeras), metodología empleada (Experimental y no experimental) y disciplinas como puede observarse en la Tabla I.

En cuanto a la disciplina, los artículos fueron agrupados en cuatro categorías: Investigaciones de áreas Básicas o de Soporte; Investigaciones en área Clínica; Investigaciones en Educación y Formación Odontológica y Estudios Epidemiológicos.

Para su análisis se seleccionaron variables y dimensiones de la siguiente manera:

I) Revisión por un comité de ética: A) expresamente menciona que fue evaluado, revisado y aprobado por un comité de Bioética y B) no hace ninguna mención al respecto.

2) Consentimiento informado: A) indica expresamente haberlo realizado y describe detalles acerca del procedimiento efectuado (texto del documento e información verbal que el paciente debe conocer y asentir), B) sólo menciona que el paciente firmó el consentimiento informado sin puntualizar la manera empleada para llevarlo a cabo y C) no se menciona el consentimiento informado.

3) Justificación del estudio: A) el artículo explicita los beneficios de la investigación y el factor riesgo - beneficio, B) no está explicito, pero se deduce de la lectura del texto, C) no está explicitado ni describe alguna información orientadora para deducirlo.

4) Selección de la muestra: A) detalla de qué manera se realizó, B) o no lo describe.

Además se revisó las normas de publicación o instrucciones a los autores, respecto de la existencia o no de requisitos éticos.

Para el registro de datos se utilizó una planilla en Excel donde se dispusieron las variables y se efectuó una categorización de las mismas.

\section{Resultados}

Las variables analizadas presentan valores que se describen a continuación:

Revisión por un Comité de Bioética: el $89 \%$ de los artículos analizados no expresa haber sido sometido a evaluación por una comisión a fin como se observa en la Tabla II. Consentimiento Informado: el $68 \%$ de la muestra total no hace ninguna mención acerca de la realización del Consentimiento Informado. Un $21 \%$ de los autores menciona haberlo realizado pero no detalla qué información se suministró ni la manera como encaró este procedimiento.

Justificación del estudio en seres humanos: Esta característica estudiada responde al Informe Belmont cuando hace alusión al principio de beneficencia que expresa "incrementar al máximo los beneficios y reducir al mínimo los riesgos que pudieran ocurrir en la investigación. El 91\% de los estudios analizados explica con detalles que la investigación está plenamente justificada, y se explaya sobre los beneficios que se obtendrán con los hallazgos y que los mismos superan ampliamente los riesgos.

La selección de la muestra: es otra de las variables utilizadas para el análisis de los aspectos bioéticos. La misma, al igual que la anterior, responde al Informe Belmont en lo que se refiere 
al Principio de Justicia que expresa "...la exigencia moral de procedimientos y resultados justos en la selección de los sujetos de investigación...”, “...la selección de los sujetos demanda que los investigadores sean equitativos, imparciales, o sea que no ofrezcan la investigación potencialmente benéfica sólo a algunos pacientes a los que favorecen ni se seleccionen sólo personas "indeseables" para investigaciones riesgosas..."

Los resultados arrojaron que el $67 \%$ de los artículos, explican con detalle la metodología utilizada para seleccionar a los sujetos de investigación, poniendo de manifiesto de esta manera que se respetó el principio de justicia seleccionando a los sujetos como la metodología lo requiere, adquiriendo un tratamiento igualitario para todos los individuos. El $23 \%$, si bien no expresa con detalles la metodología utilizada para la selección de los sujetos, se ha observado que presentan una información, que aunque acotada, hace presumir que no se ha vulnerado el principio de justicia.

Normas para autores: Esta cualidad ha sido incorporada al análisis de este trabajo para conocer el perfil de las revistas científicas respecto a las normativas que cada una de ellas adopta y que expresamente están manifestadas como requisito a cumplimentar por los autores para que su publicación pueda ser admitida.

En el $67 \%$ de las revistas analizadas no se advierte una normativa expresa respecto a los aspectos bioéticos que deben ser observados para su publicación.

Análisis de la variable "Normas para Autores" Se ha estudiado la asociación de la misma con las variables independientes: a) Período, b) Procedencia, c) Metodología empleada y d) formato.

Como puede observarse en el grafico I, ambos Periodos tienen valores similares. Así el $\mathrm{I}^{\circ}$ periodo (2000 - 2005), arrojo un resultado del $28 \%$ de artículos científicos que estaban publicados en revistas que expresan requerimientos bioéticos en las instrucciones para autores y el $2^{\circ}$ periodo (2006 - 2010), un $38 \%$. Se aplicó la prueba del chi cuadrado de Pearson, no hallándose diferencia estadísticamente significativa en la asociación de "periodo" y "normas para autores".

Del mismo modo se analizo la variable Metodología empleada hallándose un $29 \%$ de artículos publicados en revistas que exigen requisitos bioéticos para su aceptación y que emplearon la metodología experimental, y un 36\% que utilizaron la no experimental. No se encontraron diferencias estadísticamente significativas en la asociación de estas variables (Normas para autores - Metodología empleada) en la aplicación de la prueba del chi cuadrado de Pearson.

La variable Procedencia sí mostro valores diferentes. Así se encontró que solo el $4 \%$ de las los textos analizados en revistas argentinas exigían el cumplimiento de los aspectos bioeticos en las normas para autores. Mientras que el $64 \%$ de las revistas extranjeras analizadas, expresaban entre las normas para autores, requerimientos bioeticos. La prueba del chi cuadrado de Pearson arrojo un valor $p$ 0,000 I, demostrando una diferencia estadística altamente significativa en la asociación de ambas cualidades (Normas para autores - Procedencia).

El análisis de la variable Formato de las revistas arrojó los siguientes resultados: el $5 \%$ de los artículos en formato impreso pertenecían a revistas que expresamente solicitaban requerimientos bioeticos para la publicación, mientras que los revisados en formato electrónico representaron el $65 \%$, como puede observarse en el grafico I. Estas variables (Normas para autores Formato) demostraron estar asociadas ya que la prueba del chi cuadrado de Pearson aplicada dio un valor $p$ 0,000I, es decir con una diferencia estadística altamente significativa.

\section{Discusión}

Un número importante de trabajos de investigación $(89 \%)$ en la muestra total no manifiesta que el mismo haya sido evaluado por un órgano competente. Esta característica está plasmada en la Declaración de Helsinki que aconseja que “....antes de realizar el estudio, el protocolo debe enviarse para consideración, comentario, consejo y aprobación, a un comité de ética de investigación..."

Así mismo, en el total de la muestra, el 32\% de los trabajos revisados expresan haber realizado el Consentimiento Informado, explicando con detalles la manera que se utilizó para llevar adelante el proceso del $\mathrm{Cl}$ y en otros casos solo mencionándolo sin dar precisiones, es decir de aquellos recaudos y procedimientos por el cual 


\begin{tabular}{|c|c|c|c|c|c|c|c|c|}
\hline \multicolumn{3}{|c|}{ PERIODOS } & \multicolumn{2}{|c|}{ PROCEDENCIA } & \multicolumn{2}{|c|}{$\begin{array}{l}\text { METODOLOGÍA } \\
\text { EMPLEADA }\end{array}$} & \multicolumn{2}{|c|}{ FORMATO } \\
\hline & $2000-2005$ & $2006-2010$ & ARG. & EXTRANJ. & EXPERIM. & $\begin{array}{c}\text { NO } \\
\text { EXPERIM. }\end{array}$ & IMPRESO & ELEC. \\
\hline $\mathrm{n}$ & 50 & 50 & 50 & 50 & 45 & 55 & 39 & 61 \\
\hline
\end{tabular}

Tabla I. Distribución de la muestra.

\begin{tabular}{|c|c|c|c|c|c|c|c|c|c|c|c|c|c|}
\hline $\mathbf{n}$ & REV. CÉ & \multicolumn{3}{|c|}{ CI } & \multicolumn{3}{|c|}{ JUST } & \multicolumn{3}{c|}{ SE M } & \multicolumn{3}{|c|}{ N A } \\
\hline & SI & NO & D & M & NO & E & T & NO & P & SD & NO & E & NO \\
\hline 100 & $11 \%$ & $89 \%$ & $11 \%$ & $21 \%$ & $68 \%$ & $91 \%$ & $8 \%$ & $1 \%$ & $67 \%$ & $26 \%$ & $7 \%$ & $33 \%$ & $67 \%$ \\
\hline
\end{tabular}

Tabla 2. Tabla de frecuencias de las variables estudiadas en la muestra total.

\begin{tabular}{|c|c|c|c|}
\hline \multicolumn{4}{|l|}{ Referencias } \\
\hline $\begin{array}{l}\text { REV. CE: Revisión por un } \\
\text { Comité de Bioética }\end{array}$ & SI - Lo expresa & NO - No lo expresa & \\
\hline $\begin{array}{l}\text { Cl: Consentimiento } \\
\text { Informado }\end{array}$ & D - describe el procedimiento & M-Solo lo menciona & NO - ni menciona ni describe \\
\hline JUST: Justificación & E - Está explicito & $\mathrm{T}$ - Se deduce del texto & $\begin{array}{l}\text { NO - no está explicito ni se } \\
\text { deduce del texto }\end{array}$ \\
\hline $\begin{array}{l}\text { SE M: Selección de la } \\
\text { muestra }\end{array}$ & P - Puntualiza & SD - Se Deduce & $\begin{array}{l}\text { NO - no está explicito ni se } \\
\text { deduce del texto }\end{array}$ \\
\hline NA: Normas para Autores & $\begin{array}{l}\text { E - Expresa requerimientos } \\
\text { éticos }\end{array}$ & $\begin{array}{l}\text { NO - No Expresa } \\
\text { requerimientos éticos }\end{array}$ & \\
\hline
\end{tabular}

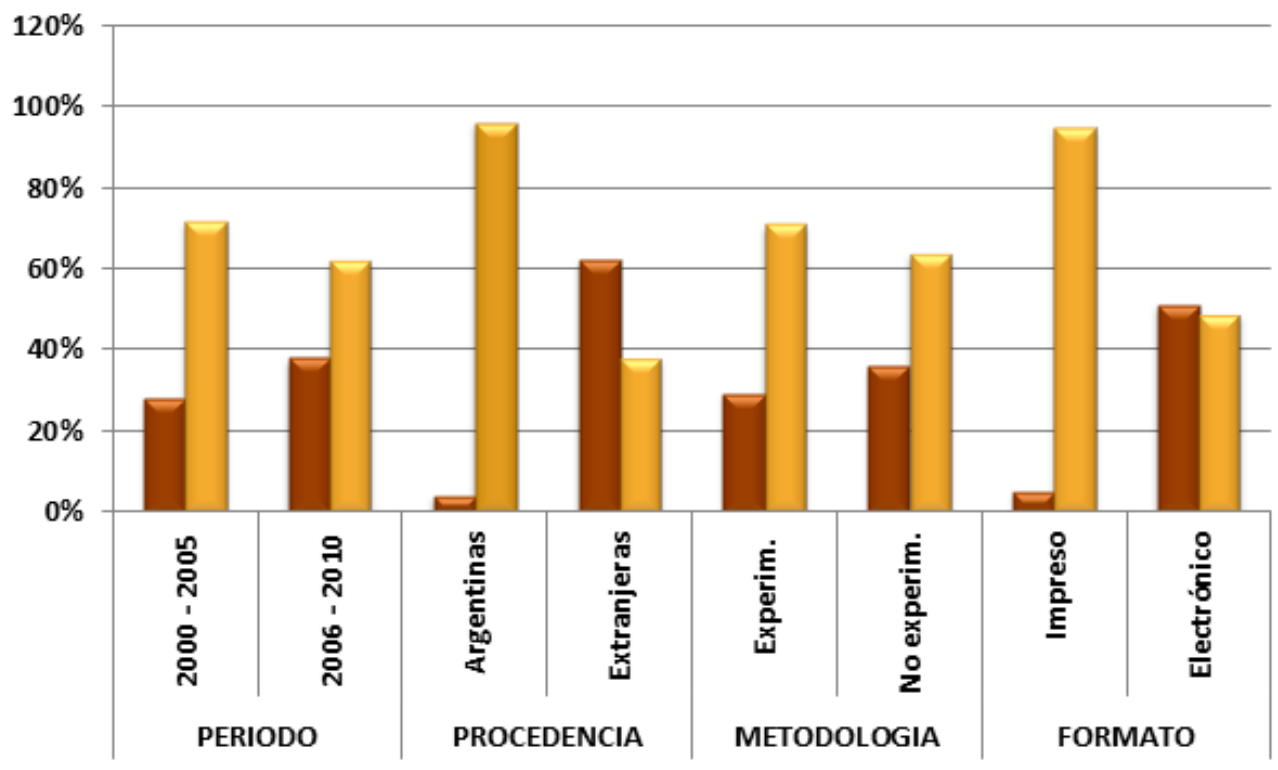

Q Revistas que exigen requerimientos bioéticos en las Normas para Autores $\square$ Revista que NO exigen requerimientos bioéticos en las Normas para Autores

Grafico I. Análisis de la variable "Normas para Autores". 
se explica al individuo que participa en una investigación sobre todos los aspectos de la misma, como la metodología en la cual va a ser sometido, beneficios, riesgos, tratamientos alternativos etc. invitándolo a firmar su aceptación a formar parte de la investigación, una vez recibida y comprendida toda la información suministrada.

Esta variable fue utilizada para el análisis de los aspectos bioéticos, debido a que es una recomendación de suma importancia en todos los documentos de Bioética. Así, la Declaración de Helsinki expresa “...y cada uno de estos debe recibir información adecuada sobre objetivos, métodos, financiamiento, afiliaciones institucionales, beneficios calculados, riesgos previsibles y todo aspecto pertinente de la investigación. Luego de lo cual debe prestar el consentimiento informado...".

Mancini Rueda y Lolas Stepke ${ }^{15}$ realizaron un trabajo cuyo objetivo general fue determinar el cumplimiento explícito de requisitos bioéticos básicos por parte de los investigadores que realizan estudios científicos en seres humanos y establecer si existen criterios éticos incorporados en la evaluación de los trabajos que son presentados para su publicación en una muestra representativa de las principales revistas científicas de la Región, indexadas en las bases bibliográficas LILACS y MEDLINE. Uno de los hallazgos de este estudio fue que entre un 46 y $49 \%$ de los textos revisados de ensayos clínicos, señalaban el Consentimiento Informado y haber sido sometido a un Comité evaluador de los aspectos bioéticos, mientras que los estudios no farmacológicos analizados representaron entre un $27 \%$ y $32 \%$ respecto a estas variables $(\mathrm{Cl}$ y Revisión por un Comité de Bioética).

En el análisis de la variable "Normas para Autores" se ha observado un mayor número de revistas extranjeras que expresan requisitos bioéticos entre las instrucciones para autores, respecto a los resultados hallados en las argentinas (4\%), siendo esta diferencia estadísticamente significativa como se explico más arriba. Se puede inferir que la Argentina aun no se ha desarrollado en la investigación científica odontológica comparativamente con otros países y por consiguiente en los aspectos bioéticos.

Trajano Sardenberg y colaboradores ${ }^{16}$ en su trabajo, demostraron que las revistas que hacen referencia a los aspectos éticos en la investigación representan el $20,8 \%$.

\section{Conclusiones}

Los resultados de este estudio no deben interpretarse que los aspectos éticos no fueron tenidos en cuenta, sino que analiza el fenómeno de la exteriorización de los mismos en la publicación.

En la investigación odontológica, se observa que si bien el Código de Ética puntualiza los aspecto bioéticos a tenerse en cuenta como así también las pautas, sugerencia y consejos establecidos en los documentos que fueron descriptos arriba, no se observa que estén reflejados en los trabajos difundidos, como así también no están comprendidas en las políticas editoriales de las revistas.

Es importante acotar que en la Información para los Autores de la Revista de la Facultad de Odontología de la Universidad Nacional del Nordeste, esta explicita la exigencia de la evaluación de aspectos bioéticos en estos términos: "Cuando se describan investigaciones en seres humanos o con animales de laboratorio, la revista exigirá la mención del comité de ética que aprobó el protocolo de investigación y la institución responsable".

Trajano Sardenberg y colaboradores concluían al respecto "La comparación con nuestros resultados, en que solamente $20,8 \%$ de las revistas se refieren de alguna manera a la ética de la investigación en seres humanos, demuestra que las publicaciones brasileñas están poco involucradas en este debate".

Pensamos que los requerimientos bioeticos, tal cual están expresados en los documentos, deben estar incluidos en el texto del artículo, por tal motivo este trabajo tiende a reflexionar sobre la necesidad de explicitar los aspectos bioéticos en toda investigación en seres humanos, para que sea fuertemente interiorizada por la comunidad científica que los consulta. 
INVESTIGACIÓN

Acción del óleo calcáreo en el tratamiento

de las queilitis por contacto crónicas.

\section{Bibliografía}

I. Martínez-Otero Pérez V. Temas de nuestro tiempo, Psicología, Educación, Sociedad y Calidad de Vida. Madrid: Ed. Fundamentos; 1999.

2. Breña Sánchez J., Breña Sánchez G., Breña Sánchez R. Ética y valores I. México: Ed. Esfinge; 2005.

3. Taylor S, Bogdan R. Introducción a los métodos cualitativos de investigación social: la búsqueda de significados. Madrid: Ed. Paidós; 1992.

4. García-Tamayo F, Reyes-García MG. Las buenas y las malas costumbres del investigador. Invest. clín. [en línea]. Sept. 2006, vol.47, no.3 [cited 23 June 2007], p.2/5-218.[consulta Julio 2007] http://www2.scielo.org.ve/ scielo. php?script=sci_arttext\&pid=S0535$5|33200600030000| \& \mid n g=e n \& n r m=i s o>$. ISSN 0535-5I33.

5. Código de Nüremberg. (Traducción: Gonzalo Herranz) [consulta: Julio 2007] www.unav.es/ humbiomedicas/deontologiaBiol/nuremberg. pdf

6. Bunge, M. Ética y Ciencia. 3ra. ed. Buenos Aires: Siglo XX; 1983.

7. Calva Rodríguez, R. México D.F.: Ed. McGraw Hill: 2006. p 3.

8. León Correa, FJ. Fundamentos y Principios de Bioética Clínica, Institucional y Social. Acta bioeth. [revista en la Internet]. 2009 [citado 2010 Sep2]; 15 (I): 7078. Disponible en: http://www.scielo.cl/ scielo.php?script=sci_arttext\&pid $=\mathrm{S} \mid 726$ 569X2009000100009\&Ing=es. doi: 10.4067/ SI726-569X2009000100009.

9. Sgreccia, E. Manual de Bioética. Italia: Ed. Diana; 1996. p. 533.

10. Herranz G. Experimentación científica en el hombre. Capítulo 17 de Deontología Biológi- ca. Facultad de Medicina, Ciencias y Farmacia. Universidad de Navarra. En http://www.unav. es/cdb/dbcapol 7a.html [acceso 201/ 9 oct 24]

II. Mandó L. Investigaciones biomédicas en seres humanos. Cuestiones jurídicas y bioéticas en torno a la Muerte. Investigaciones biomédicas en seres humanos: "Ciencia vs. Utilidad social". URL http://muerte.bioetica.org/mono/mono30. htm. [acceso 2009 agos 24].

12. Gracia D. Investigación Clínica. En: Consentimiento Informado (Separata) y en Profesión médica, Investigación y Justicia Sanitaria. Sta. Fe de Bogotá: Ed. El Búho; 1998.

13. Vélez Correa LA. Ética Médica. Corporación para Investigaciones Biológicas. Colombia: 1996. p. 165.

14. Sardenberg Trajano, Müller SS, Pereira Hamilton R, Oliveira RA, Hossne William S. Análisis de los aspectos éticos de la investigación en seres humanos contenidos en las instrucciones a los autores de 139 revistas científicas brasileñas. Acta bioeth. [revista en la Internet]. 2000 Dic [citado 2010 Sep 2]; 6(2): 293-307. Disponible en: http://www.scielo. $\mathrm{cl} /$ scielo.php?script $=$ sci_arttext\&pid $=$ SI 726 569X2000000200008\&lng=es. doi: 10.4067/ S1726-569X2000000200008.

15. Mancini Rueda R, Lolas Stepke F. Evaluación Bioética de trabajos de investigación en seres humanos publicados en América Latina y el Caribe. Acta bioeth. [revista en la Internet]. 200I [citado 2012 Mar 2I]; 7(I): 159-169. Disponible en: http://www.scielo. $\mathrm{cl} /$ scielo.php?script $=$ sci_arttext\&pid $=\mathrm{SI}$ 726. $569 \times 2001000100012 \&$ Ing=es. http://dx.doi. org/I0.4067/SI 726-569X200I000I000I2.

16. Sardenberg Trajano, Müller SS, Pereira Hamilton R, Oliveira RA, Hossne William S. Op. Cit. 